\title{
Energy Relations for Plane Waves Reflected from Moving Media
}

\author{
Daly, P.; Gruenberg, Harry
}

Published in:

Journal of Applied Physics

Link to article, DOI:

$10.1063 / 1.1709154$

Publication date:

1967

Document Version

Publisher's PDF, also known as Version of record

Link back to DTU Orbit

Citation (APA):

Daly, P., \& Gruenberg, H. (1967). Energy Relations for Plane Waves Reflected from Moving Media. Journal of Applied Physics, 38(11), 4486-4489. https://doi.org/10.1063/1.1709154

\section{General rights}

Copyright and moral rights for the publications made accessible in the public portal are retained by the authors and/or other copyright owners and it is a condition of accessing publications that users recognise and abide by the legal requirements associated with these rights.

- Users may download and print one copy of any publication from the public portal for the purpose of private study or research.

- You may not further distribute the material or use it for any profit-making activity or commercial gain

- You may freely distribute the URL identifying the publication in the public portal

If you believe that this document breaches copyright please contact us providing details, and we will remove access to the work immediately and investigate your claim. 
$0.2 \mu$ in silicon the change in refractive index is of order of $10^{-3}$. Such a variation would cause the reflection coefficient to be quite small until the light became nearly parallel to the slip plane. Then appreciable reflection could occur, accounting for the strong anisotropic scattering observed when light is incident parallel to the nominal slip plane direction.

\section{ACKNOWLEDGMENTS}

The authors wish to acknowledge the advice and support of Robb Thomson, T. A. Davis for assistance in the electrical measurements, A. Montgomery for obtaining the infrared scattering results, and Texas Instruments for supplying germanium as requested.

TJOURNAL OF APPLIED PHYSICS

VOLUME 38 , NUMBER 11

OCTOBER 1967

\title{
Energy Relations for Plane Waves Reflected from Moving Media
}

\author{
P. DalY* AND Harry Gruenberg $\dagger$ \\ Laboratory of Electromagnetic Field Theory, The Technical University of Denmark, Lyngby, Denmark
}

(Received 10 April 1967; in final form 6 July 1967)

\begin{abstract}
When a plane wave is obliquely incident from vacuum on a semi-infinite moving medium, the energy flow carried by the incident wave, is in general, not carried away by the reflected and transmitted waves. This is only the case when the medium velocity is parallel to its vacuum interface. Otherwise there is a net inflow or outflow of electromagnetic energy, which can be accounted for by the change of stored energy in the system, and the work done by the mechanical forces acting on the medium. A detailed energy balance is drawn up for two different media moving normal to their vacuum interfaces: (a) a moving conductor and (b) a moving dielectric.
\end{abstract}

\section{INTRODUCTION}

The problem of plane-wave reflection from a moving mirror has been treated by Sommerfeld ${ }^{t}$ who showed how the frequency of the reflected wave is altered by the mirror. More recently, the reflection and transmission of plane waves in a moving dielectric has been considered. ${ }^{2}$ In this case, the dependence of the reflected and transmitted wave frequencies and wave vectors was derived in terms of the frequency of the incoming wave, the permittivity, and the velocity of the dielectric. It was claimed that the reflection and transmission coefficients derived from the Poynting vectors associated with the fields, added to unity independent of the direction of the medium velocity. When the moving medium, be it conductor or dielectric, moves parallel to its own interface with vacuum, this conclusion is justified. However, when the medium has a velocity component normal to its interface, the rate of change of stored energy in the system and the rate of mechanical work done must be taken into account.

First, the simpler problem of reflection of an obliquely incident plane wave from a moving conductor is dealt with, and subsequently, the analogous problem for a moving dielectric. In both cases, the problem is solved in the reference frame of the observer ${ }^{3}$ rather than in the proper frame of the medium. This has the advantage

\footnotetext{
* Research Fellow on a grant from Science Research Council, London, U.K.

$\dagger$ On leave from Syracuse University on a Fulbright Research Grant.

1 A. Sommerfeld, Optics (Academic Press Inc., New York, 1964).

${ }_{2}$ C. Yeh, J. Appl. Phys. 36, 3513 (1965).

3 V. P. Pyati, J. Appl. Phys. 38, 652 (1967).
}

that the method will also work when more than one dielectric is involved. An energy balance based on Minkowski's theory of electrodynamics is drawn up to demonstrate that the energy density carried by the incident wave is not totally carried away by the reflected and transmitted waves. Reflection and transmission coefficients are derived and, for the case of normal wave incidence on the moving dielectric, plotted as a function of velocity. We use the notation of Ref. 2 where appropriate.

\section{MOVING CONDUCTOR}

We assume that at time $t=0$, a perfect conductor occupies the half-space, $z<0$. The conductor is supposed to have a constant velocity $v$ along the positive $z$ axis. The electric field component of the incoming plane wave is taken along the $y$ axis. The geometry of the problem is shown in Fig. 1. The frame of reference is that of an observer who sees the conductor moving with velocity $v$ perpendicular to its surface. The incident and reflected waves seen by this observer may be written as

$$
\begin{aligned}
E_{y}{ }^{i} & =E_{0} \exp \left(i k_{x} x+i k_{z} z-i \omega t\right), \\
\omega B_{x}{ }^{i} & =-k_{z} E_{y}{ }^{i}, \\
\omega B_{z}^{i} & =k_{x} E_{y}{ }^{i},
\end{aligned}
$$

where

$$
\begin{gathered}
k_{z}=-k_{0} \cos \theta_{0}, \quad k_{x}=k_{0} \sin \theta_{0} \quad k_{0}=\omega / c, \\
E_{y}{ }^{r}=E_{r} \exp \left(i k_{x}{ }^{r} x-i k_{z}{ }^{r} z-i \omega^{r} t\right), \\
\omega^{r} B_{x}{ }^{r}=k_{z}{ }^{r} E_{y}{ }^{r}, \\
\omega^{r} B_{z}{ }^{r}=-k_{x}{ }^{r} E_{y}{ }^{r},
\end{gathered}
$$


where

$$
\left(k_{x}^{r}\right)^{2}+\left(k_{z}\right)^{2}=\left(\omega^{r} / c\right)^{2} .
$$

There are, of course, no fields in the perfect conductor. The boundary condition on the fields demands the continuity of tangential $\mathbf{E}+\boldsymbol{\nabla} \times \mathbf{B}$ at $z=v t$. Therefore, we find that

$$
\begin{aligned}
& {\left[1-\left(v k_{z} / \omega\right)\right] E_{0} \exp \left[i k_{x} x+i\left(v k_{z}-\omega\right) t\right]} \\
& \quad+\left[1+\left(v k_{z}^{r} / \omega^{r}\right)\right] E_{\tau} \exp \left[i k_{x}^{r} x-i\left(v k_{z}^{r}+\omega^{r}\right) t\right]=0,
\end{aligned}
$$

for all $x$ and $t$. It may be concluded that

$$
k_{x}=k_{x}{ }^{r}=k_{0} \sin \theta_{0} \quad-v k_{z}{ }^{r}-\omega^{r}=v k_{z}-\omega .
$$

From Eqs. (4) and (6), we deduce that

where

$$
\begin{aligned}
& \omega^{r}=\omega \gamma^{2}\left(1+\beta^{2}+2 \beta \cos \theta_{0}\right) \\
& k_{z}{ }^{r}=-k_{0} \gamma^{2}\left[2 \beta+\left(1+\beta^{2}\right) \cos \theta_{0}\right],
\end{aligned}
$$

Equation (5) then becomes

$$
E_{r}=-\left[\left(1+\beta^{2}+2 \beta \cos \theta_{0}\right) /\left(1-\beta^{2}\right)\right] E_{0} .
$$

We now consider the energy balance in the reflection process. 'To this end, a cylinder of unit cross section is erected whose axis is parallel to the $z$ axis and which contains a portion of the interface. The normal component of the Poynting vector on the top face of the cylinder associated with the incident wave is

$S_{z}{ }^{i}=-\frac{1}{2} E_{y}{ }^{i} H_{x}{ }^{i *}=-\left(\cos \theta_{0} / 2 c \mu_{0}\right) E_{0}{ }^{2}=-c U_{0} \cos \theta_{0}$,

where $U_{0}$ is the energy density in the incident wave given by

$$
U_{0}=\left(1 / 2 c^{2} \mu_{0}\right) E_{0}^{2}
$$

Similarly,

$$
S_{z}{ }^{r}=-\frac{1}{2} E_{y}{ }^{r} H_{x}{ }^{*}=\frac{2 \beta+\left(1+\beta^{2}\right) \cos \theta_{0}}{1+\beta^{2}+2 \beta \cos \theta_{0}} \frac{E_{r}{ }^{2}}{2 c \mu_{0}} .
$$

The total rate at which electromagnetic energy leaves the above cylinder is

$$
\begin{aligned}
P_{\theta l}=S_{z}{ }^{i}-S_{z}^{r} & =\left[2 \beta /\left(1-\beta^{2}\right)^{2}\right] \\
& \times\left[\left(1+\beta \cos \theta_{0}\right)^{2}+\left(\beta+\cos \theta_{0}\right)^{2}\right] c U_{0},
\end{aligned}
$$

since the side surfaces do not give any net contribution. As the conductor moves along the $z$ axis, the energy stored in the fields changes at a rate proportional to the conductor velocity. Thus, the rate of decrease of stored energy in the field is

$$
\begin{aligned}
P_{s} & =(v / 2)\left(\mathbf{E}^{i} \cdot \mathbf{D}^{i *}+\mathbf{E}^{r} \cdot \mathrm{D}^{r *}\right) \\
& =\left[2 \beta /\left(1-\beta^{2}\right)^{2}\right]\left[\left(1+\beta \cos \theta_{0}\right)^{2}+\beta^{2}\left(\beta+\cos \theta_{0}\right)^{2}\right] c U_{0} .
\end{aligned}
$$

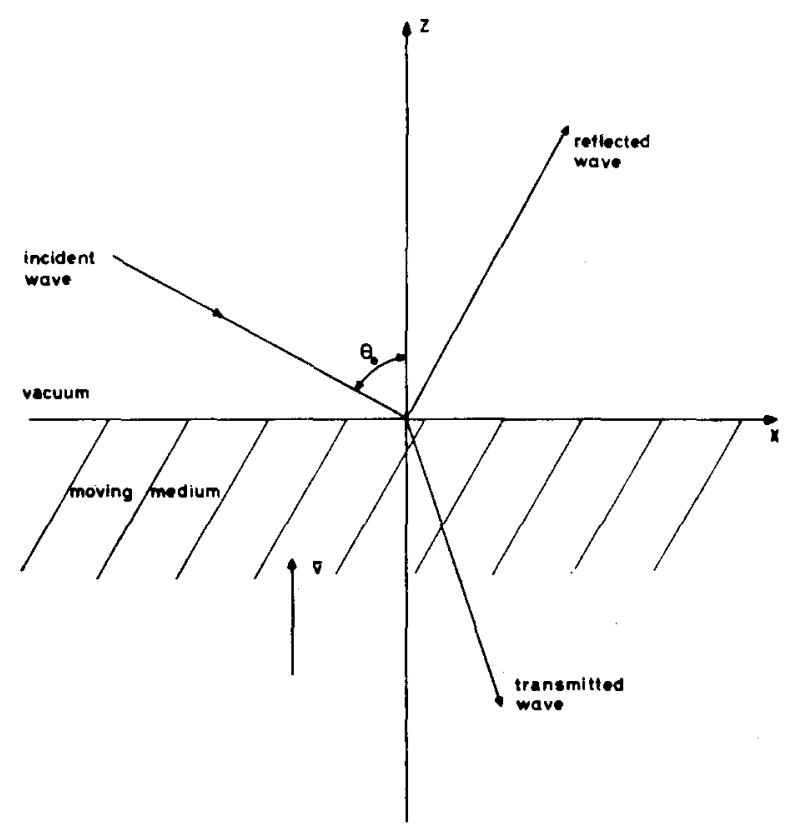

FiG. 1. Geometry of the problem.

This decrease in stored energy does not completely account for the excess of radiated energy. The difference must be supplied by the mechanical power required to keep the conductor moving at a uniform velocity against the radiation pressure.

From Eqs. (13) and (15), the rate at which mechanical work is to be supplied is given by

$$
P_{m}=P_{e l}-P_{s}=\left[2 \beta\left(\beta+\cos \theta_{0}\right)^{2} /\left(1-\beta^{2}\right)\right] c U_{0} .
$$

This conclusion may easily be verified by a direct calculation of the radiation pressure $\mathbf{F}$ exerted by the field on the conductor. This may be deduced from the stress-energy tensor but comes more directly from the following argument. The surface current density induced on the conductor is given by

$$
\begin{aligned}
\mathbf{K} & =\mathbf{n} \times(\mathbf{H}-\mathbf{v} \times \mathbf{D}), \\
K_{y} & =H_{x}-v D_{y} \\
& =\left(2 E_{0} / c \mu_{0}\right)\left(\beta+\cos \theta_{0}\right) .
\end{aligned}
$$

The pressure acting on the surface of the conductor is simply

$$
\begin{aligned}
\mathbf{F} & =\frac{1}{4} \mathbf{K} \times \mathbf{B}^{*}=-\frac{1}{4} K_{y} B_{x}{ }^{*} \\
& =-\left[\left(\beta+\cos \theta_{0}\right)^{2} /\left(1-\beta^{2}\right)\right] 2 U_{0} .
\end{aligned}
$$

As expected, the required mechanical input power, $-\mathrm{F} \cdot \nabla$, is indeed equal to $P_{m}$ given by Eq. (16). The energy balance has thus been completely accounted for. The mechanical work supplied and the decrease in stored energy explain the excess of reflected energy over the incident energy. Without the externally applied mechanical energy, the conductor will decelerate under the action of the radiation force. It is also clear from 
the preceding arguments that, if the conductor only has a tangential velocity, the force on its surface can do no work and the time-averaged stored energy remains constant. Only in this case will the reflected and incident energy flows be equal, i.e., the reflection coefficient be unity. The reflection coefficient, as defined in Ref. 2, is given by

$$
R=\frac{\left[2 \beta+\left(1+\beta^{2}\right) \cos \theta_{0}\right]\left(1+\beta^{2}+2 \beta \cos \theta_{0}\right)}{\left(1-\beta^{2}\right)^{2} \cos \theta_{0}} .
$$

\section{MOVING DIELECTRIC}

The geometry of the problem is again as in Fig. 1, where the medium occupying the region $z<0$ is taken to be a moving dielectric. In the moving dielectric, $E_{y}$ satisfies the wave equation transformed ${ }^{4}$ to the moving frame

$$
\begin{aligned}
\left\{\nabla^{2}-1 / c^{2}\left(\partial^{2} / \partial t^{2}\right)-\left[\left(n^{2}-1\right)\right.\right. & \left.\gamma^{2} / c^{2}\right] \\
& \left.\times(\partial / \partial t+\mathrm{v} \cdot \nabla)^{2}\right\} E_{y}=0,
\end{aligned}
$$

where

$$
n^{2}=\epsilon_{1} / \epsilon_{0} .
$$

The plane-wave solution in the moving dielectric is

$$
\begin{aligned}
E_{y}{ }^{t} & =E_{t} \exp \left(i k_{x}{ }^{t} x+i k_{z}{ }^{t} z-i \omega^{t} t\right), \\
\omega^{t} B_{x}{ }^{t} & =-k_{z}{ }^{t} E_{y}{ }^{t}, \\
\omega^{t} B_{z}{ }^{t} & =k_{x}{ }^{t} E_{y}{ }^{t},
\end{aligned}
$$

where the relationship between $k_{z}{ }^{t}, k_{x}{ }^{t}$, and $\omega^{\imath}$ may be found by substituting in Eq. (20). The vacuum fields are again given by Eqs. (1) and (3). The boundary conditions on the moving surface require the continuity of tangential $E+\nabla \times B$ and $H-\nabla \times D$. The first condition gives

$$
\begin{aligned}
{[1-} & \left.\left(v k_{z} / \omega\right)\right] E_{0} \exp \left[i k_{x} x+i\left(v k_{z}-\omega\right) t\right] \\
& +\left[1+\left(v k_{z}{ }^{r} / \omega^{r}\right)\right] E_{r} \exp \left[i k_{x}{ }^{r} x-i\left(v k_{z}{ }^{r}+\omega^{r}\right) t\right] \\
= & {\left[1-\left(v k_{z}{ }^{t} / \omega^{t}\right)\right] E_{t} \exp \left[i k_{x}{ }^{t} x+i\left(v k_{z}{ }^{t}-\omega^{t}\right) t\right], }
\end{aligned}
$$

for all $x$ and $t$. Accordingly, we may write

$$
\begin{gathered}
k_{x}=k_{x}{ }^{r}=k_{x}{ }^{t}=k_{0} \sin \theta_{0}, \\
v k_{z}-\omega=-v k_{z}{ }^{r}-\omega^{r}=v k_{z}{ }^{t}-\omega^{t} .
\end{gathered}
$$

From these equations, we obtain for $\omega^{r}$ and $k_{z}{ }^{r}$, Eqs. (7) as before and, with the aid of Eq. (20),

where

$$
\begin{gathered}
\omega^{t}=\omega \gamma^{2}\left\{1+\beta \cos \theta_{0}-\beta Q\right\}, \\
k_{z}{ }^{t}=k_{0} \gamma^{2}\left\{\beta\left[1+\beta \cos \theta_{0}\right)-Q\right\},
\end{gathered}
$$

$$
Q^{2}=n^{2}\left(1+\beta \cos \theta_{0}\right)^{2}-\left(1-\beta^{2}\right) \sin ^{2} \theta_{0} .
$$

The second boundary condition gives

$$
\begin{aligned}
{\left[\left(k_{z} / \omega \mu_{0}\right)+v \epsilon_{0}\right] E_{0}+} & {\left[\left(-k_{z}^{r} / \omega^{r}\right)+v \epsilon_{0}\right] E_{r} } \\
& =(1 / \mu)\left[\left(k_{z}{ }^{t} / \omega^{t}\right)+\left(v / c^{2}\right)\right] E_{t} .
\end{aligned}
$$

\footnotetext{
${ }^{\star}$ K. S. H. Lee and C. H. Papas, J. Math. Phys. 5, 1668 (1964).
}

Elimination between Eqs. (23) and (27) leads to the result

$$
\begin{aligned}
& E_{r}=\left(\omega^{r} / \omega\right)\left[\left(\beta+\cos \theta_{0}-Q\right) /\left(\beta+\cos \theta_{0}+Q\right)\right] E_{0}, \\
& E_{t}=2\left(\omega^{t} / \omega\right)\left[\left(\beta+\cos \theta_{0}\right) /\left(\beta+\cos \theta_{0}+Q\right)\right] E_{0} .
\end{aligned}
$$

Expressions (7), (24), and (27) were also obtained in Ref. 2 by means of the principle of phase invariance.

Before beginning a discussion of the energy relations, we write all the necessary field expressions in the moving dielectric. From the Minkowski constitutive relations in a moving medium, ${ }^{5}$ we deduce that

$$
\begin{aligned}
& H_{x}{ }^{t}=-\frac{1}{c \mu} \frac{\beta n^{2}\left(1+\beta \cos \theta_{0}\right)-Q}{1+\beta \cos \theta_{0}-\beta Q} E_{u^{t}}, \\
& H_{z}{ }^{t}=\frac{1}{c \mu} \frac{\left(1-\beta^{2}\right) \sin \theta_{0}}{1+\beta \cos \theta_{0}-\beta Q} E_{y}{ }^{t}, \\
& D_{y^{t}}{ }=\frac{1}{c^{2} \mu} \frac{n^{2}\left(1+\beta \cos \theta_{0}\right)-\beta Q}{1+\beta \cos \theta_{0}-\beta Q} E_{y}{ }^{t} .
\end{aligned}
$$

We draw up the energy balance as previously. The total energy flow leaving the surface is given by

$$
\begin{aligned}
P_{e l}= & S_{z}{ }^{i}-S_{z}{ }^{r}-S_{z}{ }^{r} \\
= & \frac{\cos \theta_{0}}{2 c \mu} E_{0}{ }^{2}-\frac{2 \beta+\left(1+\beta^{2}\right) \cos \theta_{0}}{1+\beta^{2}+2 \beta \cos \theta_{0}} \frac{E_{r}^{2}}{2 c \mu} \\
& +\frac{\beta n^{2}\left(1+\beta \cos \theta_{0}\right)-Q}{1+\beta \cos \theta_{0}-\beta Q} \frac{E_{t}{ }^{2}}{2 c \mu} .
\end{aligned}
$$

The expression for the Poynting vector in the moving dielectric derived in Ref. 2 , is in error because it is tacitly assumed that $B_{x}=\mu_{0} H_{x}$. This is not the case in a moving medium. After some algebra, the above expression reduces to

$$
\begin{aligned}
P_{e l}=-\frac{2 \beta\left(n^{2}-1\right)\left(1+\beta \cos \theta_{0}\right)}{\left(1-\beta^{2}\right)^{2}\left(\beta+\cos \theta_{0}+Q\right)^{2}} \\
\quad\left[\left(1+\beta \cos \theta_{0}\right) M-2 \beta\left(\beta+\cos \theta_{0}\right)^{2} Q\right] c U_{0},
\end{aligned}
$$

where

$$
M=3\left(\beta+\cos \theta_{0}\right)^{2}-\left(1+\beta \cos \theta_{0}\right)^{2} .
$$

The rate of increase of stored energy as the dielectric "occupies" more free space is given by the velocity times the difference in the volume densities of stored energy in the vacuum and the dielectric:

$$
P_{s}=v\left(\frac{1}{2} \mathrm{E}_{y}{ }^{t} \cdot \mathbf{D}_{y}{ }^{i *}-\frac{1}{2} \mathrm{E}_{y}{ }^{r} \cdot \mathbf{D}_{y}{ }^{r *}-\frac{1}{2} \mathrm{E}_{y}{ }^{i} \cdot \mathbf{D}_{y}{ }^{i *}\right)
$$

On substitution from Eqs. (1), (3), (21), and (28),

${ }^{5}$ A. Sommerfeld, Electrodynamics (Academic Press Inc., New York, 1964), p. 287. 
and by means of (27), this expression reduces to

$$
\begin{aligned}
P_{s}=+ & \frac{2 \beta\left(n^{2}-1\right)\left(1+\beta \cos \theta_{0}\right)}{\left(1-\beta^{2}\right)^{2}\left(\beta+\cos \theta_{0}+Q\right)^{2}} \\
& {\left[\left(1+\beta \cos \theta_{0}\right) N-2 \beta\left(\beta+\cos \theta_{0}\right)^{2} Q\right] c U_{0}, }
\end{aligned}
$$

where

$$
N=\left(\beta^{2}+2\right)\left(\beta+\cos \theta_{0}\right)^{2}-\left(1+\beta \cos \theta_{0}\right)^{2} .
$$

Therefore, the rate at which mechanical energy has to be supplied to the system is given by the sum of Eqs. (30) and (33) and is

$$
P_{m}=-\frac{2 \beta\left(n^{2}-1\right)\left(1+\beta \cos \theta_{0}\right)^{2}\left(\beta+\cos \theta_{0}\right)^{2}}{\left(1-\beta^{2}\right)\left(\beta+\cos \theta_{0}+Q\right)^{2}} c U_{0} .
$$

Because $P_{m}$ is negative, the field is doing work on the medium. In order to stop the medium accelerating towards the incoming wave, a restraining force has to be applied to the medium to counteract the radiation pressure. We may check this conclusion by calculating the force exerted on the dielectric by the field. The volume density of force is given ${ }^{6}$ by

$$
f_{\alpha}=\left(\partial T_{\alpha \beta} / \partial x_{\beta}\right)-\partial / \partial t\left(\frac{1}{2} \mathbf{D} \times \mathbf{B}^{*}\right),
$$

where the stress tensor $T_{\alpha \beta}$ is given by

$$
T_{\alpha \beta}=\frac{1}{2} E_{\alpha} D_{\beta}{ }^{*}+\frac{1}{2} H_{\alpha} B_{\beta}{ }^{*}-\frac{1}{4} \delta_{\alpha \beta}\left(E_{\gamma} D_{\gamma}{ }^{*}+H_{\gamma} B_{\gamma}{ }^{*}\right) \text {. }
$$

When Eq. (37) is integrated over the volume of the cylinder, the force on the surface is found to be

$$
\begin{aligned}
F=T_{z z}{ }^{i} & +T_{z z}{ }^{r}-T_{z z^{t}} \\
& +(v / 2)\left(\mathrm{D}^{i} \times \mathrm{B}^{i *}+\mathrm{D}^{r} \times \mathrm{B}^{r *}-\mathrm{D}^{t} \times \mathrm{B}^{t *}\right),
\end{aligned}
$$

where

$$
T_{z z}=-\frac{1}{4} E_{y} D_{y}{ }^{*}+\frac{1}{4} H_{z} B_{z}{ }^{*}-\frac{1}{4} H_{x} B_{x}{ }^{*} .
$$

Substituting in Eq. (38) for the vacuum and dielectric fields, we conclude that

$$
\mathbf{F}=+\frac{\left(n^{2}-1\right)\left(\beta+\cos \theta_{0}\right)^{2}\left(1+\beta \cos \theta_{0}\right)^{2}}{\left(1-\beta^{2}\right)\left(\beta+\cos \theta_{0}+Q\right)^{2}} 2 c U_{0} .
$$

- W. Panofsky, M. Phillips, Classical Electricity and Magnetism (Addison-Wesley Publishing Co., Inc., Reading, Mass., 182, 1962).

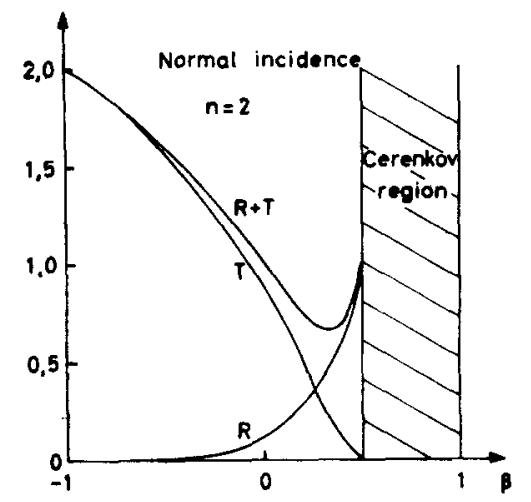

Fig. 2. Reflection and transmission coefficients for normal incidence on moving dielectric.

The field supplies work at the rate of $\mathbf{F} \cdot \nabla=-P_{m}$ in agreement with the conclusion drawn from (35). Note that the forces exerted by the field on a conductor and on a dielectric are in opposite directions.

For normal incidence, the reflection and transmission coefficients are given by

$$
\begin{gathered}
R=S_{z}{ }^{r} / S_{z}{ }^{i}=(1+\beta)^{2}(1-n)^{2} /(1-\beta)^{2}(1+n)^{2}, \\
T=S_{z}{ }^{t} / S_{z}{ }^{i}=\left[4 n /(1+n)^{2}\right]\left[(1-\beta n)^{2} /(1-\beta)^{2}\right] .
\end{gathered}
$$

The expressions $R, T$, and $R+T$ are plotted against $\beta$ in Fig. 2 for the case $n=2$. It is observed that the sum $R+T$ is only unity when $\beta=0$ or $\beta n=1$, and has a minimum when $\beta(2 n-1)=1$. At this point, the medium experiences the maximum radiation pressure, tending to draw it towards the incoming wave. When the medium travels away from the incoming wave, the transmitted energy flow is always greater than that incident upon it. This excess is provided by mechanical forces acting upon it and by the contribution from the stored energy change. Finally, there is a cut-off at $\beta n=1$. At greater velocities, the Cerenkov condition in the dielectric is satisfied, and we may no longer assume homogeneous plane waves to propagate in the dielectric. 